\title{
Gender-Related Differences in Hypertrophic Cardiomyopathy: A Systematic Review and Meta-Analysis
}

\author{
Angkawipa Trongtorsak ${ }^{a}$ Natchaya Polpichai ${ }^{b}$ Sittinun Thangjui ${ }^{c}$ \\ Jakrin Kewcharoen ${ }^{d}$ Ratdanai Yodsuwan $^{c}$ Amrit Devkota $^{a}$ \\ Harvey J. Friedman ${ }^{\text {e }}$ Alfonso Q. Estrada ${ }^{f}$ \\ anternal Medicine Residency Program, AMITA Health Saint Francis Hospital, Chicago, IL, USA; baculty of Medicine \\ Songklanagarin Hospital, Prince of Songkla University, Songkhla, Thailand; ' Internal Medicine Residency Program, \\ Bassett Healthcare Network, New York, NY, USA; dinternal Medicine Residency Program, University of Hawaii,

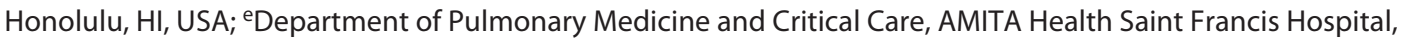 \\ Chicago, IL, USA; fDepartment of Cardiovascular Medicine, AMITA Health Saint Francis Hospital, Chicago, IL, USA
}

\section{Keywords}

Hypertrophic cardiomyopathy · Gender-related differences · Mortality $\cdot$ Heart failure

\begin{abstract}
Background: Gender-related differences in phenotypic expression and outcomes have been established in many cardiac conditions; however, the impact of gender in hypertrophic cardiomyopathy (HCM) remains unclear. We conducted a systematic review and meta-analysis to assess the differences in clinical outcomes between female and male HCM patients. Methods: We searched MEDLINE and EMBASE from inception to October 2020. Included were cohort studies that compared outcomes of interest including all-cause mortality, HCM-related mortality, and worsening heart failure (HF) or HF hospitalization between male and female. Data from each study were combined using the random effects model to calculate pooled odds ratio (OR) with $95 \%$ confidence interval $(\mathrm{Cl})$. Results: Eleven retrospective cohort
\end{abstract}

studies with a total of 9,427 patients (3,719 females) were included. Female gender was significantly associated with an increased risk of all-cause mortality (pooled $\mathrm{OR}=1.63$, $95 \%$ Cl: $1.26-2.10, p \leq 0.001$ ), HCM-related mortality (pooled $\mathrm{OR}=1.47,95 \% \mathrm{Cl}: 1.08-2.01, p=0.015)$, and worsening $\mathrm{HF}$ or $\mathrm{HF}$ hospitalization (pooled $\mathrm{OR}=2.05,95 \% \mathrm{Cl}$ : 1.76-2.39, $p \leq 0.001$ ). Conclusions: Female gender was associated with a worse prognosis in $\mathrm{HCM}$. These findings suggest the need for improved care in women including early identification of disease and more possible aggressive management. Moreover, gender-based strategy may benefit in HCM patients.

(c) 2021 S. Karger AG, Basel

\section{Introduction}

Hypertrophic cardiomyopathy (HCM) is the most common genetic cardiac disorder worldwide with the prevalence of $0.2 \%$ in the general population [1]. It is caused by sarcomere protein gene mutation character- 
ized by left ventricular wall thickening [2]. Clinical presentations range from asymptomatic to angina, heart failure (HF), or even sudden cardiac death [3].

Gender-related difference in phenotypic expression and outcomes is now an evolving topic and has been established in many cardiac conditions. Previous studies revealed that $\mathrm{HCM}$ was male predominant; the ratio of men to women was around 3:2 [4-6]. Also, women were older at diagnosis and tended to be symptomatic later in life compared with men $[6,7]$. However, the clinical outcomes comparing females and males are still unclear. Some studies reported that women had a worse prognosis [8-10]. On the other hand, some studies reported no differences regarding the rates of mortality $[11,12]$. Thus, we conducted a systematic review and meta-analysis to assess the differences in clinical outcomes including allcause mortality, HCM-related mortality, and worsening HF or HF hospitalization between women and men with HCM.

\section{Methods}

\section{Search Strategy}

Two investigators (N.P. and R.Y.) independently performed a systematic search of the MEDLINE and EMBASE from inception to October 2020 using a search strategy including the terms "hypertrophic cardiomyopathy," "familial hypertrophic cardiomyopathy," "hypertrophic obstructive cardiomyopathy," "gender," "female," and "male," as described in see online suppl. File 1 (see www.karger.com/doi/10.1159/000517618 for all online suppl. material). We hand-searched the bibliographies of selected studies and meta-analyses to identify further eligible studies. Only full articles in English were included.

\section{Inclusion Criteria}

The eligible criteria included the following: (1) cohort studies (prospective or retrospective), case-control studies, experimental studies, or randomized controlled trials that were conducted in HCM patients comparing outcomes of interest including all-cause mortality, HCM-related mortality, or worsening HF or HF hospitalization between females and males were included. (2) Relative risk, odds ratio (OR), hazard ratio, incidence ratio with $95 \%$ confidence intervals (CIs), or sufficient raw data to calculate for effect size must be provided.

Data Extraction and Quality Assessment Tool

Two authors (A.D. and S.T.) independently extracted the data from included studies, with disputes resolved by consensus following discussion with a third author (A.T.). Included observational studies were assessed using the Newcastle-Ottawa Scale (NOS) for quality assessment [13]. The NOS was used to assess each study's quality. The NOS uses a star system (0-9) to evaluate included studies in 3 domains: recruitment and selection of the participants, similarity and comparability between the groups, and ascertainment of the outcome of interest among cohort and case-control studies.
Higher scores represent higher study quality. The Cochrane collaboration tool for assessing risk of bias was used to evaluate the quality of each randomized controlled trial by assessing as a judgment (high, low, or unclear) for individual elements from 5 domains (selection, performance, attrition, reporting, and other) [14].

\section{Definitions}

HCM was defined as the thickness of nondilated left ventricle $\geq 13-15 \mathrm{~mm}$ by 2 -dimensional echocardiography or cardiac magnetic resonance in adults in absence of other cardiac or other systemic etiology that can lead to ventricular hypertrophy [15]. HCMrelated mortality was defined as death due to following: (1) sudden cardiac death, (2) death due to refractory HF, (3) stroke-related death [16].

\section{Statistical Analysis}

This meta-analysis was performed using random effects model. The extracted studies were excluded from the analysis if they did not justify an outcome in each cohort. The effect size was used to determine the difference of outcomes between the 2 groups. Qstatistic and $I^{2}$ statistic were used to assess evidence of heterogeneity [17]. The $I^{2}$ statistic ranges in value from 0 to $100 \%\left(I^{2}<25 \%\right.$, low heterogeneity; $I^{2}=25-50 \%$, moderate heterogeneity; and $I^{2} \geq$ $50 \%$, substantial heterogeneity) [18]. Publication bias was assessed using a funnel plot, Begg's test, and Egger's test $[19,20]$. The $p$ value $<0.05$ in publication bias tests was suggestive of publication bias. Sensitivity analysis was also performed to assess the influence of individual studies on overall meta-analysis, as described by Patsopoulos et al. [17,21]. All analyses were conducted using STATA software (version 14 STATA Corp., College Station, TX, USA).

\section{Results}

\section{Literature Search}

The initial literature search identified 2,885 studies from MEDLINE and EMBASE. We excluded 526 studies due to duplication. A total of 2,358 studies were excluded as they were not cohort studies, case-control studies, experimental trials, or randomized controlled trials, or did not study the population of interest. Thirty-two studies were relevant for evaluation. Twenty-one studies were excluded due to reasons given in the PRISMA flow diagram in Figure 1. Therefore, 11 studies were included in this meta-analysis [11, 12, 22-30].

\section{Description of Included Studies}

A total of 11 retrospective cohort studies from 2018 to October 2020 were included in this meta-analysis. The final analysis included a total of 9,427 patients $(3,719$ women, 5,708 men) with a range of follow-up from 2 to 13 years. The pooled mean age was $55.2 \pm 14.6$ and 48.9 \pm 13.6 in females and males, respectively. The characteristics of the included studies are shown in Table 1. 


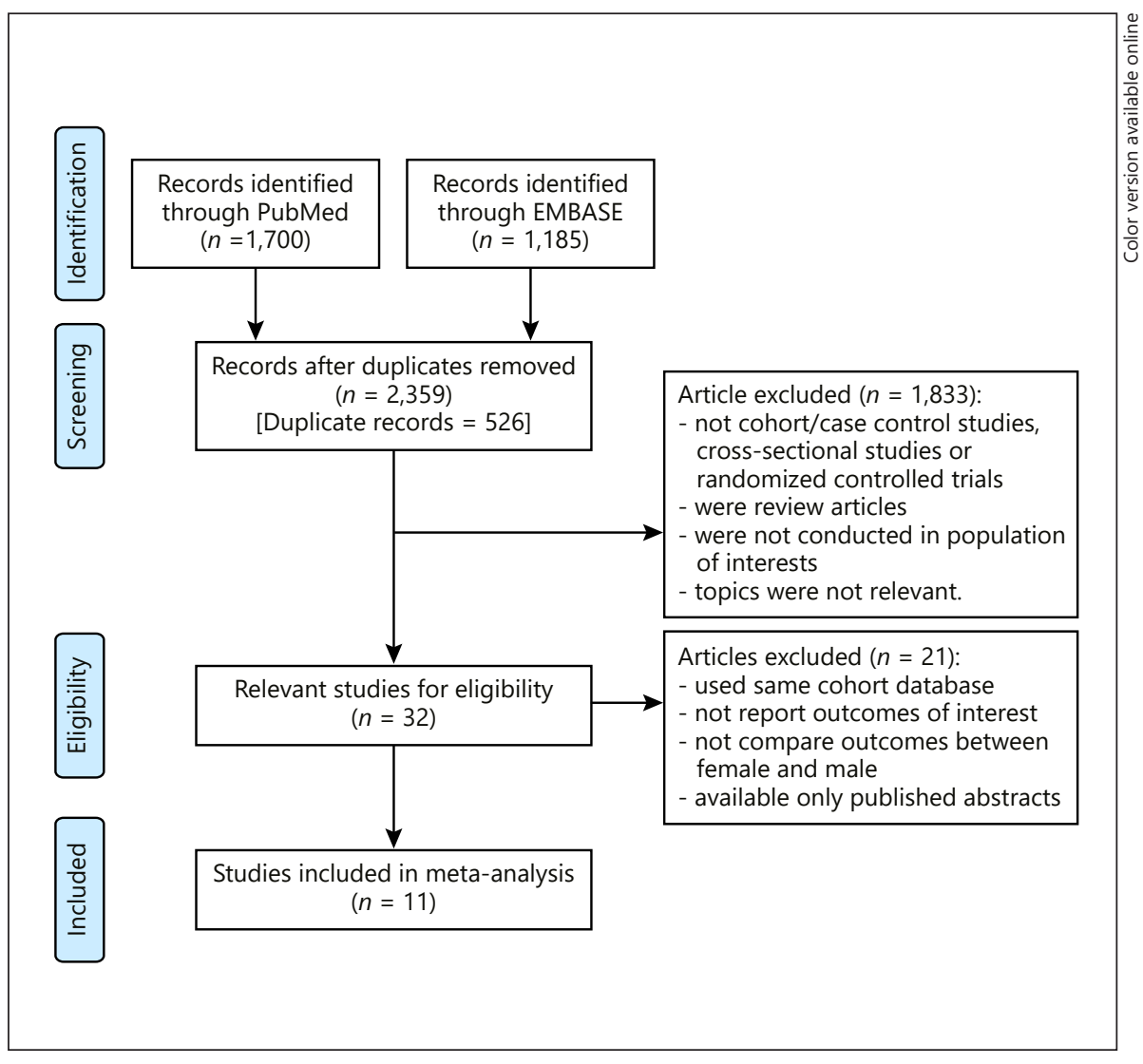

Fig. 1. PRISMA flow diagram illustrating study selection process.

\section{Quality Assessment Tool}

The NOS was used for all 11 observational studies to assess the study quality. The NOS uses a star system from 0 to 9 to evaluate 3 domains including selection, comparability, and outcomes. Higher scores represent higher study quality. NOS of included studies are described in online suppl. File 2.

\section{Meta-Analysis Results}

All-Cause Mortality

Outcomes regarding all-cause mortality were available in 7 studies [11, 12, 24, 25, 27, 28, 30]. Female gender was associated with a higher risk of all-cause mortality (pooled $\mathrm{OR}=1.63,95 \%$ CI: $\left.1.26-2.10, p \leq 0.001, I^{2}=35 \%\right)$. The forest plot is shown in Figure 2.

HCM-Related Mortality

Outcomes regarding HCM-related mortality were available in 10 studies [11, 12, 22-24, 26-30]. Female gender was significantly associated with an increased risk of HCM-related mortality (pooled OR $=1.47,95 \%$ CI: 1.08 $\left.2.01, p=0.015, I^{2}=43 \%\right)$. The forest plot is shown in Figure 3 .

\section{Worsening HF or HF Hospitalization}

Outcomes regarding worsening HF or HF hospitalization were available in 7 studies $[11,12,22,25,28-30]$. Female gender was associated with worsening HF or HF hospitalization than male gender (pooled OR $=2.05,95 \%$ CI: $\left.1.76-2.39, p \leq 0.001, I^{2}=0 \%\right)$. The forest plot is shown in Figure 4.

\section{Sensitivity Analysis}

We conducted a sensitivity analysis for each outcome by excluding 1 study at a time to assess the stability of the results of the meta-analysis. For every outcome, none of the results was significantly changed. As the results were similar to that of the main meta-analysis, it indicated that our results were robust.

\section{Publication Bias}

We investigate the effect of potential publication bias on HCM-related mortality by creating a funnel plot from included studies. The vertical axis of the funnel plot represents study size (standard error of log OR) while the horizontal axis represents effect size (log OR). The funnel plot is shown in online suppl. Figure 1. We also per-
40

Pulse 2021;9:38-46 DOI: $10.1159 / 000517618$
Trongtorsak et al. 


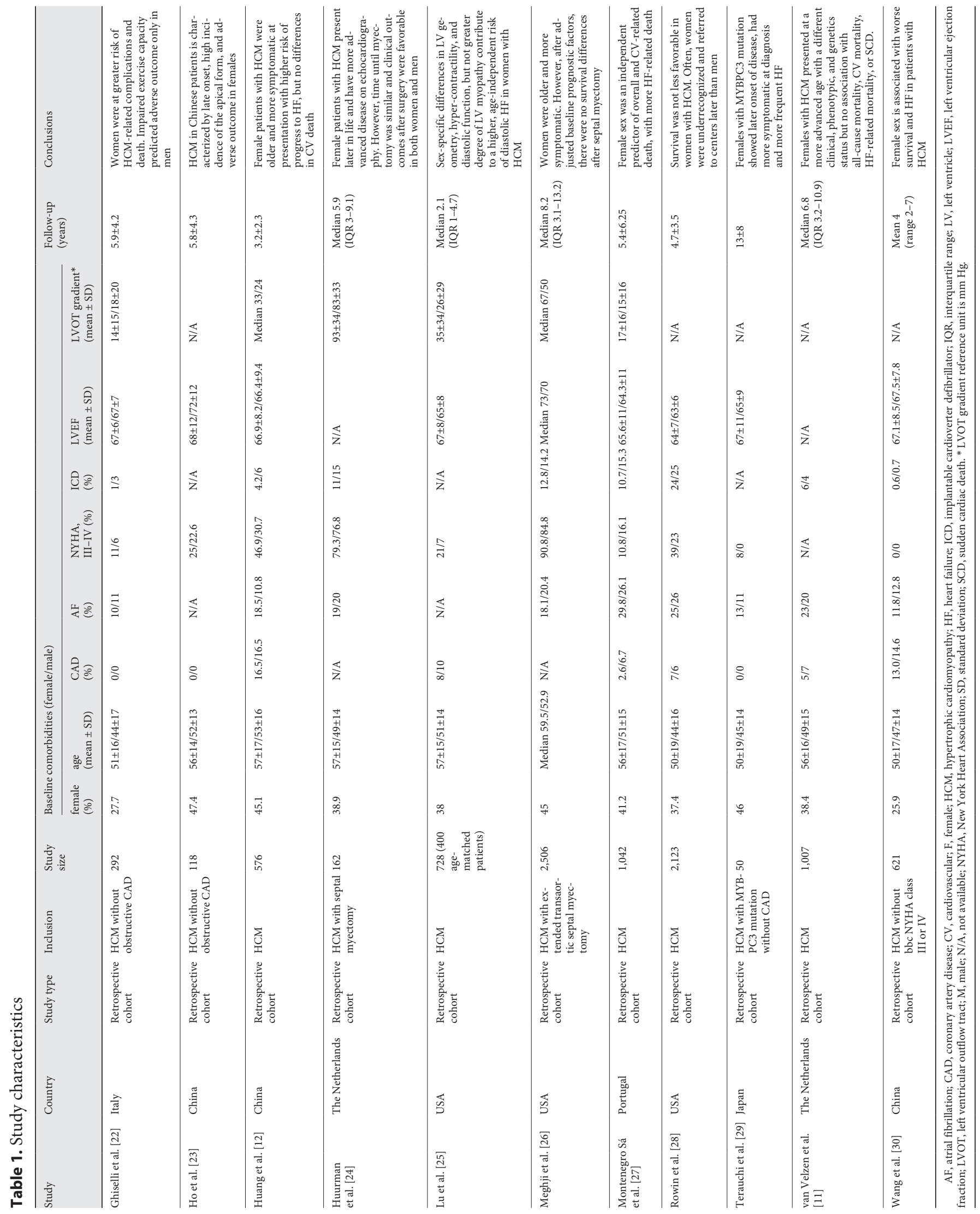




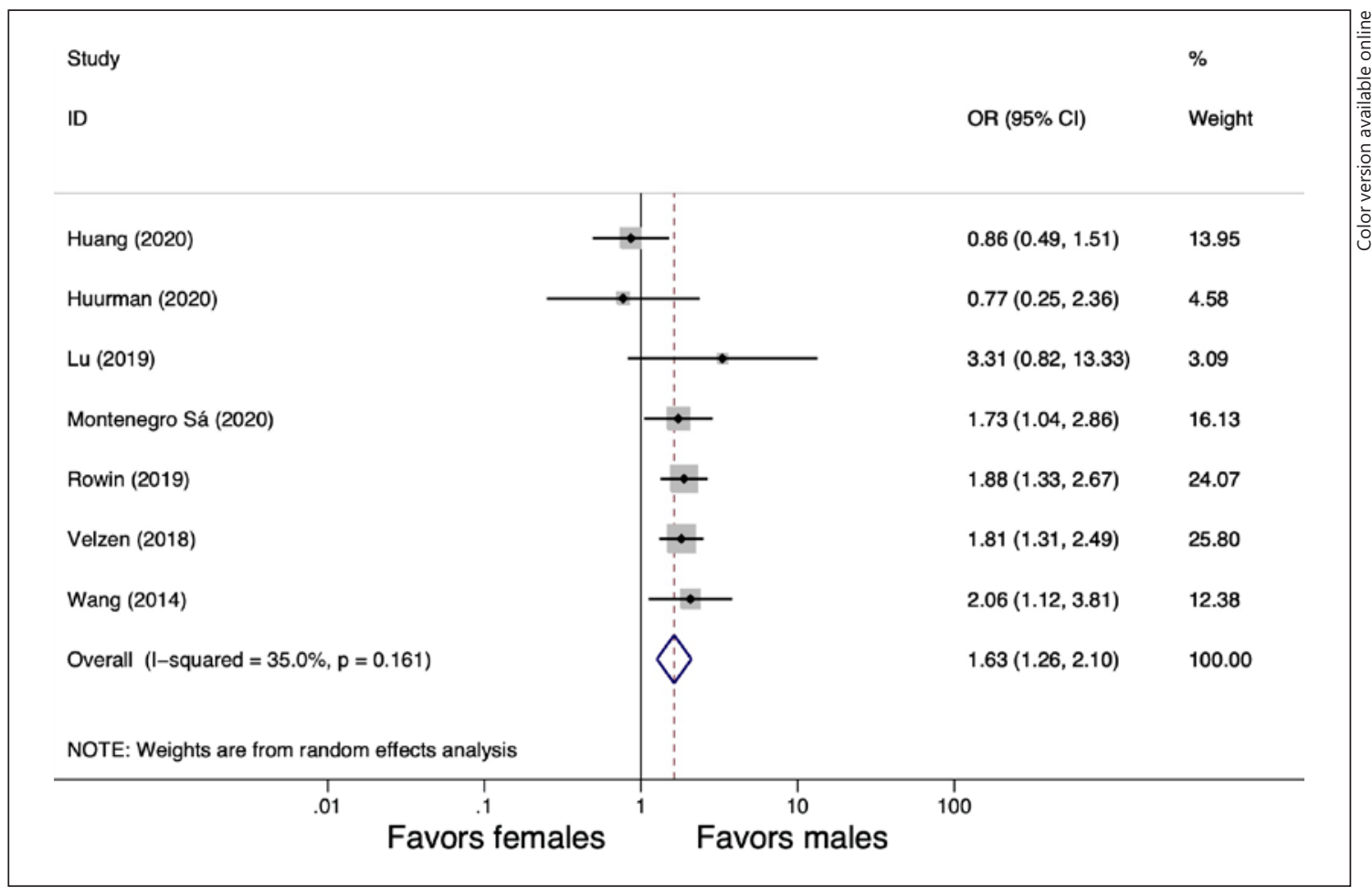

Fig. 2. Forest plot for pooled gender differences in all-cause mortality.

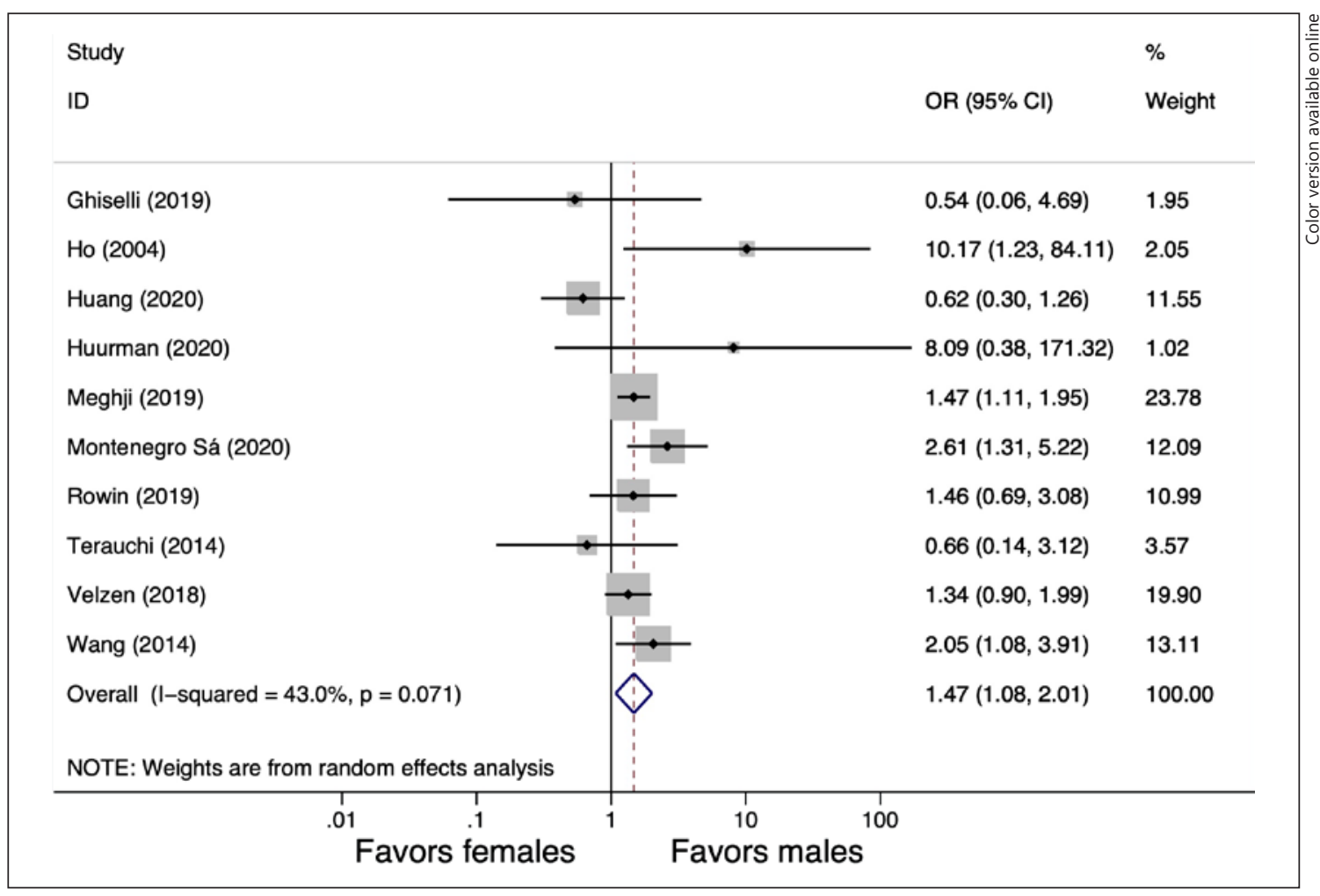

Fig. 3. Forest plot for pooled gender differences in HCM-related mortality. HCM, hypertrophic cardiomyopathy. 


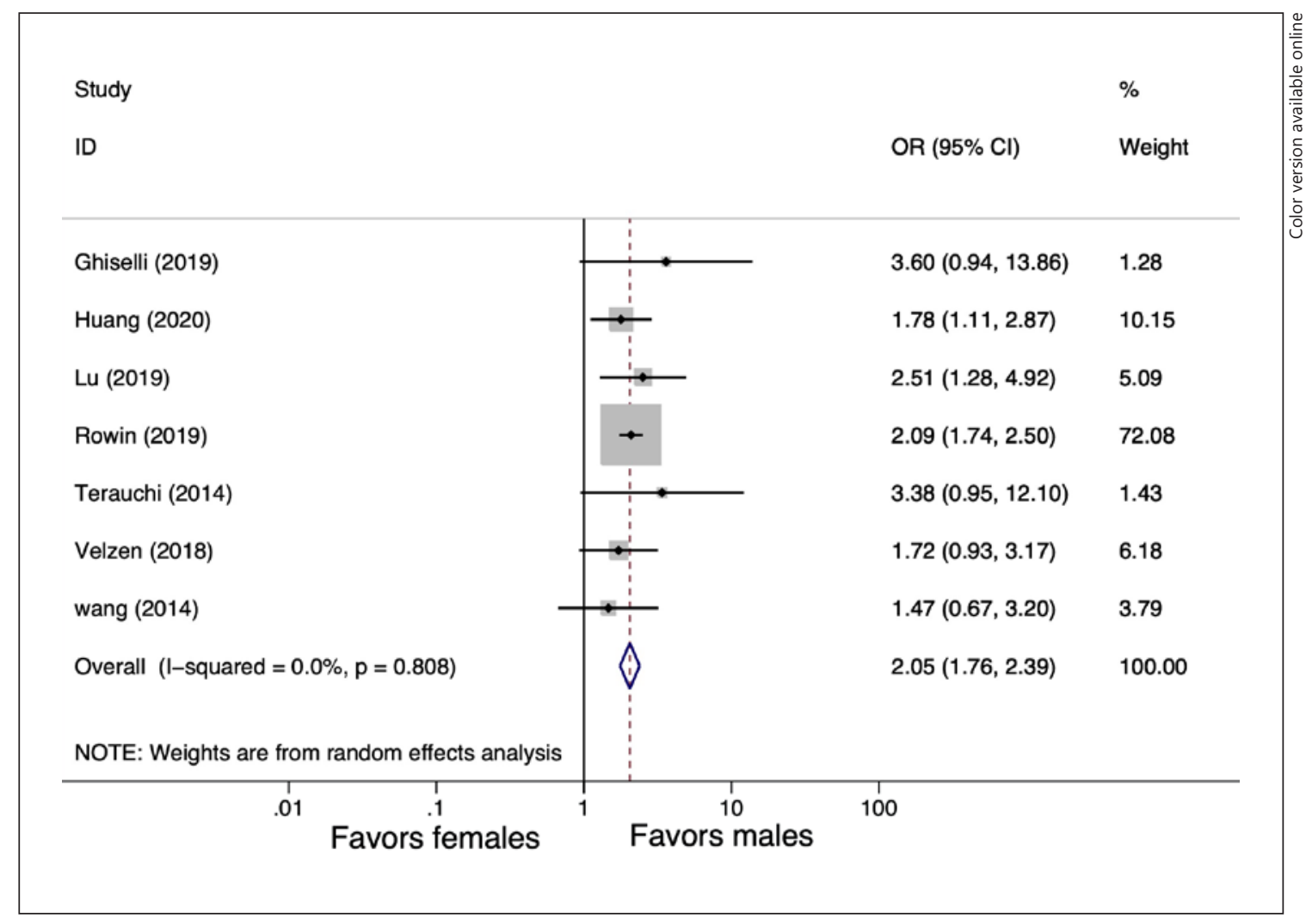

Fig. 4. Forest plot for pooled gender differences in worsening HF or HF hospitalization. HF, heart failure.

formed Begg's and Egger's test to assess publication bias. There was no publication bias according to the Begg's and Egger's test (Begg's test, $p=0.283$ and Egger's test; $p=$ 0.144 ).

Of the 11 studies reviewed, only 7 studies provided outcomes regarding all-cause mortality and worsening HF or HF hospitalization. Thus, it is insufficient to reject the assumption of no funnel plot asymmetry. Therefore, we did not perform a funnel plot, Begg's test, or Egger's test $[31,32]$.

\section{Discussion}

Previous studies reported the existence of gender-related difference in HCM [4-6]; nevertheless, the differences of prognosis between female and male remain unclear. Thus, we conducted this systematic review and meta-analysis to assess the clinical outcomes between women and men with HCM. The primary end points in this study are (1) all-cause mortality, (2) HCM-related mortality, and (3) worsening HF or HF hospitalization.
Our findings indicated that female gender in HCM patients was associated with a worse prognosis including higher rates of all-cause mortality, HCM-related mortality, and worsening HF or HF hospitalization.

Studies have shown the influences of gender in expression and outcomes in patients with HF. Females were found to have more prevalence of HF with preserved ejection fraction $[33,34]$. On the other hand, males tended to have higher rates of HF with reduced ejection fraction and coronary artery disease [33, 3537]. Moreover, female gender was found to have higher survival rates. Framingham Heart Study showed that female patients with HF had significantly higher survival rates [38]. Gerber et al. [39] also reported that men had higher rates of cardiovascular deaths. Interestingly, Adam et al. [40] reported that women with nonischemic cardiomyopathy had higher survival rates; however, there were no differences in mortality when the etiology was ischemic cardiomyopathy. It is possible that the higher rates of mortality in males could be from higher rates of $\mathrm{HF}$ with reduced ejection fraction and ischemic cardiomyopathy. Our meta-analysis showed 
that female gender was associated with a worse prognosis in HCM. Compared with studies in HF, most HCM studies excluded patients with coronary artery disease. Also, most HCM patients had preserved EF. These may explain the differences of outcomes from previous studies focused on HF. However, further studies are needed to find out why female gender was associated with a worse outcome.

Postmenopausal endocrine changes in women may play an important role in the difference of presentations and outcomes in HCM between males and females. Estrogen is believed to be involved in several pathways of cardiac diastolic functions [41, 42]. Studies also showed that deficiency of estrogen was associated with impaired ventricular relaxation, myocardial hypertrophy, and fibrosis $[41,42]$. Our studies found that females with HCM were diagnosed, on average, at the age of menopause. It can be hypothesized that estrogen deficiency can lead to worsening symptoms and progression of disease. Nevertheless, further studies focusing on postmenopausal changes and outcomes in females are needed to support this hypothesis.

Age could be 1 of the confounders. Women were significantly older than men at the time of diagnosis and follow-up in most included studies. We were unable to analyze data with an adjusted age due to the absence of raw data. The studies by Geske et al. and Montenegro Sá et al. $[9,27]$ found that female gender was an independent factor for all-cause mortality even after adjusting for age. The study by Janget al. [10] also found that female gender was associated with higher HF hospitalization and the composite of HF and CV death after adjusted effect size. Moreover, Lu et al. [25] reported that women were found to have higher incidence of worsening HF after age match. However, some studies revealed there were no gender differences in mortality after age adjustment. Huang et al. [12] showed that gender did not influence mortality rates. Rowel et al. [28] found that female gender was not associated with higher mortality after adjusting for age. Our meta-analysis found that female gender was associated with a worse prognosis; however, the results were not adjusted for age. Thus, further studies with adjusted age or other comorbidities are warranted to confirm our findings.

Evidence showed that women were more symptomatic at the time of diagnosis and progressed to advanced disease than men. Women were also found to have more obstructive physiology $[4,9,26]$. The progression of symptoms and clinical course may be explained by a higher prevalence of left ventricular outflow tact obstruc- tion in females. However, Meghji et al. [26] reported that even though women had more symptoms, there were no outcome differences between female and male gender after surgical myectomy. Thus, a poor outcome in women could be from a delay in diagnosis and treatment. Also, $\mathrm{HCM}$ is an autosomal genetic disorder. In theory, women and men should have equal prevalence. Nonetheless, many studies reported a marked male predominance [46]. This finding could be from the underdiagnosis of female HCM. As delayed diagnosis may cause female patients to be denied timely standard treatment options such as ICD for primary prevention or surgical septal reduction, this can lead to advanced disease and higher mortality; indeed, we should focus on the improvement of care in women including early diagnosis and treatment.

\section{Limitations}

We acknowledge some limitations in our meta-analysis. First, this meta-analysis was based on observational studies with reported imbalance in baseline characteristics. This carries a risk of confounding bias. Further studies with better baseline adjustment are needed to support our hypothesis. Second, some studies did not primarily look at the outcomes of interest. This can cause bias to analysis due to incomplete data reported in each study.

\section{Conclusions}

Previous studies reported that female gender was older and more symptomatic at time of diagnosis. Moreover, our meta-analysis showed that women were associated with a worse prognosis including higher rates of mortality and worsening HF or HF hospitalization. These findings suggest the need for early diagnosis of female patients and possible gender-based strategy for diagnosis and treatment.

\section{Acknowledgement}

We thank Eleanor Truex, Chicago Metro Regional Medical Librarian, AMITA Health Saint Francis Hospital.

\section{Statement of Ethics}

Ethical review and approval were not required as the study is based exclusively on published literature.
Trongtorsak et al. 


\section{Conflict of Interest Statement}

There are no conflicts of interest to disclose.

\section{Funding Sources}

This research did not receive any specific grant from funding agencies in the public, commercial, or not-for-profit sectors.

\section{Author Contributions}

Conceived and designed the analysis: A.T., S.T., J.K., and A.F. Collected the data: A.T., S.T., N.T., J.K., R.Y., and A.D. Performed the analysis: A.T. and S.T. Wrote the paper: A.T., S.T., J.K., and N.T. Critical revision of the article: H.F. and A.Q.E.

\section{References}

1 Maron BJ, Gardin JM, Flack JM, Gidding SS, Kurosaki TT, Bild DE. Prevalence of hypertrophic cardiomyopathy in a general population of young adults. Echocardiographic analysis of 4111 subjects in the CARDIA Study. Coronary artery risk development in (young) adults. Circulation. 1995;92(4):785-9.

2 Klues HG, Schiffers A, Maron BJ. Phenotypic spectrum and patterns of left ventricular hypertrophy in hypertrophic cardiomyopathy: morphologic observations and significance as assessed by two-dimensional echocardiography in 600 patients. J Am Coll Cardiol. 1995; 26(7):1699-708.

3 Maron BJ, McKenna WJ, Danielson GK, Kappenberger LJ, Kuhn HJ, Seidman CE, et al. American college of cardiology/European society of cardiology clinical expert consensus document on hypertrophic cardiomyopathy: a report of the American college of cardiology foundation task force on clinical expert consensus documents and the European society of cardiology committee for practice guidelines. Eur Heart J. 2003;24(21):1965-91.

4 Olivotto I, Maron MS, Adabag AS, Casey SA, Vargiu D, Link MS, et al. Gender-related differences in the clinical presentation and outcome of hypertrophic cardiomyopathy. J Am Coll Cardiol. 2005;46(3):480-7.

5 Kubo T, Kitaoka H, Okawa M, Hirota T, Hayato $\mathrm{K}$, Yamasaki N, et al. Gender-specific differences in the clinical features of hypertrophic cardiomyopathy in a community-based Japanese population: results from Kochi RYOMA study. J Cardiol. 2010;56(3):314-9.

6 Canepa M, Fumagalli C, Tini G, VincentTompkins J, Day SM, Ashley EA, et al. Temporal trend of age at diagnosis in hypertrophic cardiomyopathy: an analysis of the international sarcomeric human cardiomyopathy registry. Circ Heart Fail. 2020;13(9):e007230.

7 Dimitrow PP, Czarnecka D, Jaszcz KK, Dubiel JS. Sex differences in age at onset of symptoms in patients with hypertrophic cardiomyopathy. J Cardiovasc Risk. 1997;4(1):33-5.

8 Klarich KW, Attenhofer Jost CH, Binder J, Connolly HM, Scott CG, Freeman WK, et al. Risk of death in long-term follow-up of patients with apical hypertrophic cardiomyopathy. Am J Cardiol. 2013;111(12):1784-91.

9 Geske JB, Ong KC, Siontis KC, Hebl VB, Ackerman MJ, Hodge DO, et al. Women with hy- pertrophic cardiomyopathy have worse survival. Eur Heart J. 2017;38(46):3434-40.

10 Jang JH, Shin SH, Beak YS, Ko KY, Kwon SW, Park SD, et al. Impact of gender on heart failure presentation in non-obstructive hypertrophic cardiomyopathy. Heart Vessels. 2020; 35(2):214-22.

11 van Velzen HG, Schinkel AFL, Baart SJ, Huurman R, van Slegtenhorst MA, Kardys I, et al. Effect of gender and genetic mutations on outcomes in patients with hypertrophic cardiomyopathy. Am J Cardiol. 2018;122(11): 1947-54.

12 Huang FY, Shah JP, Pu XB, Hagar A, Chen SJ. Influence of gender on clinical characteristics and outcomes in chinese patients with hypertrophic cardiomyopathy. Am J Med Sci. 2020; 360(5):517-24.

13 Wells GA, Shea B, O'Connell D, Peterson J, Welch V, Losos M, et al. The newcastle-ottawa scale (NOS) for assessing the quality of nonrandomised studies in meta-analyses. 2011. Available from: http://www.ohri.ca/ programs/clinical_epidemiology/oxford.asp.

14 Furfaro E, Nicolini L, Della Vecchia A, Di Grazia C, Raiola AM, Varaldo R, et al. Hepatitis $\mathrm{E}$ virus infection in an Italian cohort of hematopoietic stem cell transplantation recipients: seroprevalence and infection. Biol Blood Marrow Transpl. 2020;26(7):1355-62.

15 Maron BJ. Hypertrophic cardiomyopathy: a systematic review. JAMA. 2002;287(10): 1308-20.

16 Songsirisuk N, Kittipibul V, Methachittiphan $\mathrm{N}$, Charoenattasil V, Zungsontiporn N, Spanuchart I, et al. Modes of death and clinical outcomes in adult patients with hypertrophic cardiomyopathy in Thailand. BMC Cardiovasc Disord. 2019;19(1):1.

17 Higgins JP, Thompson SG. Quantifying heterogeneity in a meta-analysis. Stat Med. 2002; 21(11):1539-58.

18 Higgins JP, Thompson SG, Deeks JJ, Altman DG. Measuring inconsistency in meta-analyses. BMJ. 2003;327(7414):557-60

19 Begg CB, Mazumdar M. Operating characteristics of a rank correlation test for publication bias. Biometrics. 1994;50(4):1088-101.

20 Egger M, Davey Smith G, Schneider M, Minder C. Bias in meta-analysis detected by a simple, graphical test. BMJ. 1997;315(7109): 629.
21 Patsopoulos NA, Evangelou E, Ioannidis JP. Sensitivity of between-study heterogeneity in meta-analysis: proposed metrics and empirical evaluation. Int J Epidemiol. 2008;37(5): 1148-57.

22 Ghiselli L, Marchi A, Fumagalli C, Maurizi N, Oddo A, Pieri F, et al. Sex-related differences in exercise performance and outcome of patients with hypertrophic cardiomyopathy. Eur J Prev Cardiol. 2020;27(17):1821-31.

23 Ho HH, Lee KL, Lau CP, Tse HF. Clinical characteristics of and long-term outcome in Chinese patients with hypertrophic cardiomyopathy. Am J Med. 2004;116(1):19-23.

24 Huurman R, Schinkel AFL, de Jong PL, van Slegtenhorst MA, Hirsch A, Michels M. Impact of sex on timing and clinical outcome of septal myectomy for obstructive hypertrophic cardiomyopathy. Int J Cardiol. 2021;323:133-9.

25 Lu DY, Ventoulis I, Liu H, Kudchadkar SM, Greenland GV, Yalcin H, et al. Sex-specific cardiac phenotype and clinical outcomes in patients with hypertrophic cardiomyopathy. Am Heart J. 2020;219:58-69.

26 Meghji Z, Nguyen A, Fatima B, Geske JB, Nishimura RA, Ommen SR, et al. Survival differences in women and men after septal myectomy for obstructive hypertrophic cardiomyopathy. JAMA Cardiol. 2019;4(3):237-45.

27 Montenegro Sá F, Oliveira M, Belo A, Correia J, Azevedo O, Morais J. The sex gap in hypertrophic cardiomyopathy. Rev Esp Cardiol. 2020;73(12):1018-25.

28 Rowin EJ, Maron MS, Wells S, Patel PP, Koethe BC, Maron BJ. Impact of sex on clinical course and survival in the contemporary treatment era for hypertrophic cardiomyopathy. J Am Heart Assoc. 2019;8(21):e012041.

29 Terauchi Y, Kubo T, Baba Y, Hirota T, Tanioka K, Yamasaki N, et al. Gender differences in the clinical features of hypertrophic cardiomyopathy caused by cardiac myosin-binding protein C gene mutations. J Cardiol. 2015; 65(5):423-8.

30 Wang Y, Wang J, Zou Y, Bao J, Sun K, Zhu L, et al. Female sex is associated with worse prognosis in patients with hypertrophic cardiomyopathy in China. PLoS One. 2014;9(7): e102969.

31 Simmonds M. Quantifying the risk of error when interpreting funnel plots. Syst Rev. 2015;4:24. 
32 Debray TPA, Moons KGM, Riley RD. Detecting small-study effects and funnel plot asymmetry in meta-analysis of survival data: a comparison of new and existing tests. Res Synth Methods. 2018;9(1):41-50.

33 Lam CSP, Arnott C, Beale AL, Chandramouli C, Hilfiker-Kleiner D, Kaye DM, et al. Sex differences in heart failure. Eur Heart J. 2019; 40(47):3859-68c.

34 Beale AL, Meyer P, Marwick TH, Lam CSP, Kaye DM. Sex differences in cardiovascular pathophysiology: why women are overrepresented in heart failure with preserved ejection fraction. Circulation. 2018;138(2):198-205.

35 Cleland JG, Swedberg K, Follath F, Komajda M, Cohen-Solal A, Aguilar JC, et al. The Euroheart failure survey programmea survey on the quality of care among patients with heart failure in Europe. Part 1: patient characteristics and diagnosis. Eur Heart J. 2003;24(5): $442-63$.

36 Chang PP, Wruck LM, Shahar E, Rossi JS, Loehr LR, Russell SD, et al. Trends in hospitalizations and survival of acute decompensated heart failure in four US communities (20052014). Circulation. 2018;138(1):12-24.

37 Ho KK, Pinsky JL, Kannel WB, Levy D. The epidemiology of heart failure: the Framingham study. J Am Coll Cardiol. 1993;22(4 Suppl 1):6A-13A.

38 McKee PA, Castelli WP, McNamara PM, Kannel WB. The natural history of congestive heart failure: the Framingham study. N Engl J Med. 1971;285(26):1441-6.

39 Gerber Y, Weston SA, Enriquez-Sarano M, Berardi C, Chamberlain AM, Manemann SM, et al. Mortality associated with heart failure after myocardial infarction: a contemporary community perspective. Circ Heart Fail. 2016;9(1):e002460.

40 Adams KFJr, Dunlap SH, Sueta CA, Clarke SW, Patterson JH, Blauwet MB, et al. Relation between gender, etiology and survival in patients with symptomatic heart failure. J Am Coll Cardiol. 1996;28(7):1781-8.

41 Maslov PZ, Kim JK, Argulian E, Ahmadi A, Narula N, Singh M, et al. Is Cardiac diastolic dysfunction a part of post-menopausal syndrome? JACC Heart Fail. 2019;7(3):192203

42 Zhao Z, Wang H, Jessup JA, Lindsey SH, Chappell MC, Groban L. Role of estrogen in diastolic dysfunction. Am J Physiol Heart Circ Physiol. 2014;306(5):H628-40. 